\title{
Multi-Component Integrable Systems and Invariant Curve Flows in Certain Geometries
}

\author{
Changzheng $Q U^{\dagger}$ and Junfeng SONG $\ddagger$ and Ruoxia $Y A O^{\S}$ \\ $\dagger$ Center for Nonlinear Studies, Ningbo University, Ningbo, 315211, P.R. China \\ E-mail: quchangzheng@nbu.edu.cn \\ $\ddagger$ College of Mathematics and Information Science, Shaanxi Normal University, \\ Xi'an, 710062, P.R. China \\ E-mail: songjunfeng1979@yahoo.com.cn \\ $\S$ School of Computer Science, Shaanxi Normal University, Xi'an, 710062, P.R. China \\ E-mail: rxyao@snnu.edu.cn
}

Received September 28, 2012, in final form December 27, 2012; Published online January 02, 2013 http://dx.doi.org/10.3842/SIGMA.2013.001

\begin{abstract}
In this paper, multi-component generalizations to the Camassa-Holm equation, the modified Camassa-Holm equation with cubic nonlinearity are introduced. Geometric formulations to the dual version of the Schrödinger equation, the complex CamassaHolm equation and the multi-component modified Camassa-Holm equation are provided. It is shown that these equations arise from non-streching invariant curve flows respectively in the three-dimensional Euclidean geometry, the two-dimensional Möbius sphere and $n$ dimensional sphere $\mathbb{S}^{n}(1)$. Integrability to these systems is also studied.
\end{abstract}

Key words: invariant curve flow; integrable system; Euclidean geometry; Möbius sphere; dual Schrödinger equation; multi-component modified Camassa-Holm equation

2010 Mathematics Subject Classification: 37K10; 51M05; 51B10

\section{Introduction}

Integrable systems solved by the inverse scattering method usually arise from shallow water wave, physics, optical communication and applied sciences etc. Integrable systems have many interesting properties, such as Lax-pair, infinite number of conservation laws and Lie-Bäcklund symmetries, multi-solitons, Bäcklund transformations and bi-Hamiltonian structure etc. [1,44], which are helpful to explore other properties of integrable systems $[1,44,56]$.

It is of great interest to study geometric aspects of integrable systems. So far, very few integrable systems were found to have geometric formulations. The relationship between completely integrable systems and the finite-dimensional differential geometry of curves has been studied extensively. It turns out that some integrable systems arise from invariant curve flows in certain geometries [2-7, 9-15, 21, 23, 24, 26, 28-42, 45, 47-52, 55, 57, 59, 60]. The pioneering work on this topic was done by Hasimoto [24]. He showed that the integrable nonlinear Schrödinger equation (NLS)

$$
i \phi_{t}+\phi_{s s}+|\phi|^{2} \phi=0
$$

is equivalent to the system for the curvature $\kappa$ and $\tau$ of curves $\gamma$ in $\mathbb{R}^{3}$

$$
\kappa_{t}=-2 \tau \kappa_{s}-\kappa \tau_{s}, \quad \tau_{t}=\frac{\kappa_{s s s}}{\kappa}-\frac{\kappa_{s} \kappa_{s s}}{\kappa^{2}}-2 \tau \tau_{s}+\kappa \kappa_{s}
$$

${ }^{\star}$ This paper is a contribution to the Special Issue "Symmetries of Differential Equations: Frames, Invariants and Applications". The full collection is available at http://www.emis.de/journals/SIGMA/SDE2012.html 
via the so-called Hasimoto transformation $\phi=\kappa \exp \left(i \int^{s} \tau(t, z) \mathrm{d} z\right)$. Indeed, the system (1) is equivalent to the vortex filament equation

$$
\gamma_{t}=\gamma_{s} \times \gamma_{s s}=\kappa \mathbf{b}
$$

where $\mathbf{b}$ is the binormal vector of $\gamma$. Marí Beffa, Sanders and Wang [39,51] noticed that Hasimoto transformation is a gauge transformation relating the Frenet frame $\{\mathbf{t}, \mathbf{n}, \mathbf{b}\}$ to the parallel frame $\left\{\mathbf{t}^{1}, \mathbf{n}^{1}, \mathbf{b}^{1}\right\}$. It is also a Poisson map which takes Hamiltonian structure of the NLS equation to that of the vertex filament flow [29]. The Hasimoto transformation has been generalized in [51] to the Riemannian manifold with constant curvature, which is used to obtain the corresponding integrable equations associated with the invariant non-stretching curve flows. The parallel frames and other kinds of frames are also used to derive bi-Hamiltonian operators and associated hierarchies of multi-component soliton equations from non-stretching curve flows on Lie group manifolds $[3,4,31,39]$. The KdV equation, the modified KdV equation, the SawadaKotera equation and the Kaup-Kuperschmidt equation were shown to arise from the invariant curve flows respectively in centro-equiaffine geometry [7,9,48], Euclidean geometry [21], special affine geometry $[11,35]$ and projective geometries [11,30,41].

The integrable systems with non-smooth solitary waves have drawn much attention in the last two decades because of their remarkable properties. The celebrated Camassa-Holm (CH) equation

$$
m_{t}+u m_{x}+2 u_{x} m+a u_{x}=0, \quad m=u-u_{x x}
$$

was proposed as a model for the unidirectional propagation of the shallow water waves over a flat bottom, with $u(x, t)$ representing the water's free surface in non-dimensional variables [8]. It was also found using the method of recursion operators by Fokas and Fuchssteiner [19] as a bi-Hamiltonian equation with an infinite number of conserved functionals. Geometrically, the Camassa-Holm equation arises from a non-stretching invariant planar curve flow in the centro-equiaffine geometry [9], and the periodic $\mathrm{CH}$ equation (3) describes geodesic flows on $\operatorname{diff}\left(\mathbb{S}^{1} \times \mathbb{R}\right)$ with respect to right-invariant Sobolev $H^{1}$ metric for $a=0[13,14,28]$ and BottVirasoro algebra for $a \neq 0$ [40]. A dual version of the Ito system is the two-component CamassaHolm equation [46], the periodic two-component $\mathrm{CH}$ equation also describe geodesic flows on an extended Bott-Virasoro algebra [22].

It is remarked that all nonlinear terms in the $\mathrm{CH}$ equation are quadratic. In contrast to the integrable modified $\mathrm{KdV}$ equation with a cubic nonlinearity, it is of great interest to find integrable $\mathrm{CH}$-type equations with cubic or higher-order nonlinearity and non-smooth solitary waves. To the best of our knowledge, two scalar integrable $\mathrm{CH}$-type equations with cubic nonlinearity have been discovered. The first equation is $[18,46,49]$

$$
m_{t}+\frac{1}{2}\left(\left(u^{2}+\delta u_{x}^{2}\right) m\right)_{x}=0, \quad m=u+\delta u_{x x}
$$

where $\delta= \pm 1$, and the second one is the so-called Novikov equation [25,43]

$$
m_{t}+3 u u_{x} m+u^{2} m_{x}=0, \quad m=u-u_{x x},
$$

which are completely integrable, and admit peaked solitons. Recently, systems of CH-type equations with cubic nonlinearity were also obained [20,55].

The $\mathrm{CH}$ equation can also be derived by the tri-Hamiltonian duality approach basing on bi-Hamiltonian structure of the KdV equation. Other examples of dual integrable systems obtained using the method of tri-Hamiltonian duality can be found in $[18,46]$. Nonlinear dual integrable systems, such as the $\mathrm{CH}$ equation and the modified $\mathrm{CH}$ equations, are endowed with 
nonlinear dispersion, which in most cases, enables these systems to support non-smooth solitonlike structures. It was remarked in [23] that the modified $\mathrm{CH}$ equation (4) can be regarded as a Euclidean-invariant version of the $\mathrm{CH}$ equation (3), just as the modified $\mathrm{KdV}$ equation is a Euclidean-invariant counterpart to the $\mathrm{KdV}$ equation from the viewpoint of curve flows in Klein geometries $[9,10,21,48]$.

The aim of this paper is to provide geometric formulations to multi-component integrable systems admitting non-smooth solitons. We shall show that several multi-component integrable systems with non-smooth solitons, such as a dual version of the Schrödinger equation $[17,46]$, the complex $\mathrm{CH}$ equation and multi-component modified $\mathrm{CH}$ equations arise from the invariant curve flows respectively in three-dimensional Euclidean geometry, Möbius sphere and the $n$ dimensional unit sphere $\mathbb{S}^{n}(1)$. To obtain integrable systems relating to these geometric flows, we shall use the scale limit technique. The outline of this paper is as follows. In Section 2, a non-stretching invariant binormal curve flow in $\mathbb{R}^{3}$ is introduced and studied. Making use of the system for curvature and torsion corresponding to this flow, we obtain a novel integrable Schrödinger equation by a scale limit approach, which is completely integrable system and can be obtained by the so-called tri-Hamiltonian duality approach $[18,46]$. In Section 3 , we give a brief discussion on Möbius 2-sphere $\mathrm{PO}(3,1) / H$ and the $n$-dimensional sphere $\mathrm{SO}(n+1) / \mathrm{SO}(n)$, the Cartan structure equations for curves in both geometries are reviewed, which will be used in subsequent sections to study curve flows in both geometries. In Section 4, we consider the non-stretching curve flows in Möbius 2-sphere. It is shown that the complex Camassa-Holm equation and complex Hunter-Saxton equation describe the non-stretching curve flows in Möbius 2 -sphere. The bi-Hamiltonian structure for the complex Camassa-Holm equation is obtained. In Section 5 , we study non-stretching curve flows in the $n$-dimensional sphere $\mathbb{S}^{n}(1)$. Interestingly, we find that a multi-component modified $\mathrm{CH}$ equation (a multi-component generalization of the modified Camassa-Holm equation) is equivalent to a non-stretching curve flow in $\mathbb{S}^{n}(1)$. Integrability of the system is identified. Finally, Section 6 contains concluding remarks on this work.

\section{An integrable nonlinear Schrödinger equation}

We consider the flows of space curves in $\mathbb{R}^{3}$, given by

$$
\gamma_{t}=U \mathbf{n}+V \mathbf{b}+W \mathbf{t}
$$

where $\mathbf{t}, \mathbf{n}$ and $\mathbf{b}$ denote the tangent, normal and binormal vectors of the curves, respectively. The velocities $U, V$ and $W$ depend on the curvature and torsion as well as their derivatives with respect to arc-length parameter $s$. The arc-length parameter $s$ is defined implicitly by $\mathrm{d} s=h \mathrm{~d} p$, $h=\left|\gamma^{\prime}(p)\right|$, where $p$ is a free parameter and is independent of time. We denote by $\kappa$ and $\tau$ the curvature and torsion of the curves, respectively. Governed by the flow (5), time evolutions of those geometric invariants are given by $[24,42]$

$$
\begin{aligned}
& \dot{\mathbf{t}}=\left(\frac{\partial U}{\partial s}-\tau V+\kappa W\right) \mathbf{n}+\left(\frac{\partial V}{\partial s}+\tau U\right) \mathbf{b}, \\
& \dot{\mathbf{n}}=-\left(\frac{\partial U}{\partial s}-\tau V+\kappa W\right) \mathbf{t}+\left[\frac{1}{\kappa} \frac{\partial}{\partial s}\left(\frac{\partial V}{\partial s}+\tau U\right)+\frac{\tau}{\kappa}\left(\frac{\partial U}{\partial s}-\tau V+\kappa W\right)\right] \mathbf{b}, \\
& \dot{\mathbf{b}}=-\left(\frac{\partial V}{\partial s}+\tau U\right) \mathbf{t}-\left[\frac{1}{\kappa} \frac{\partial}{\partial s}\left(\frac{\partial V}{\partial s}+\tau U\right)+\frac{\tau}{\kappa}\left(\frac{\partial U}{\partial s}-\tau V+\kappa W\right)\right] \mathbf{n}, \\
& \dot{h}=2 h\left(\frac{\partial W}{\partial s}-\kappa U\right)
\end{aligned}
$$


and

$$
\begin{aligned}
& \frac{\partial \tau}{\partial t}=\frac{\partial}{\partial s}\left[\frac{1}{\kappa} \frac{\partial}{\partial s}\left(\frac{\partial V}{\partial s}+\tau U\right)+\frac{\tau}{\kappa}\left(\frac{\partial U}{\partial s}-\tau V\right)+\tau \int^{s} \kappa U \mathrm{~d} s^{\prime}\right]+\kappa \tau U+\kappa \frac{\partial V}{\partial s} \\
& \frac{\partial \kappa}{\partial t}=\frac{\partial^{2} U}{\partial s^{2}}+\left(\kappa^{2}-\tau^{2}\right) U+\frac{\partial \kappa}{\partial s} \int^{s} \kappa U \mathrm{~d} s^{\prime}-2 \tau \frac{\partial V}{\partial s}-\frac{\partial \tau}{\partial s} V .
\end{aligned}
$$

Assuming that the flow is intrinsic, namely the arc-length does not depend on time, it implies from (6) that

$$
W_{s}=\kappa U \text {. }
$$

In terms of (7), one finds that the complex function

$$
\phi=\kappa \eta, \quad \eta=\exp \left[i \int^{s} \tau\left(s^{\prime}, t\right) \mathrm{d} s^{\prime}\right]
$$

satisfies the equation $[24,42]$

$$
\begin{aligned}
\phi_{t}= & \left(\frac{\partial^{2}}{\partial s^{2}}+|\phi|^{2}+i \phi \int^{s} \mathrm{~d} s^{\prime} \tau \bar{\phi}+\frac{\partial \phi}{\partial s} \int^{s} \mathrm{~d} s^{\prime} \bar{\phi}\right)(U \eta) \\
& +\left(i \frac{\partial^{2}}{\partial s^{2}}+i|\phi|^{2}+\phi \int^{s} \mathrm{~d} s^{\prime} \tau \bar{\phi}-i \phi \int^{s} \mathrm{~d} s^{\prime} \frac{\partial \bar{\phi}}{\partial s^{\prime}}\right)(V \eta),
\end{aligned}
$$

where $\bar{\phi}$ denotes the complex conjugate of $\phi$.

Let $U=0, V=\kappa$, where $\kappa$ is a real function, then (8) implies that $W=C_{1}$, where $C_{1}$ is a constant. Setting $C_{1}=0$, we derive from (9) the celebrated Schrödinger equation

$$
i \phi_{t}+\phi_{s s}+\frac{1}{2}|\phi|^{2} \phi=0
$$

Let $U=-\kappa_{s}, V=-\kappa \tau$, then $W=-\frac{1}{2} \kappa^{2}+C_{2}$, where $C_{2}$ is a constant. Letting $C_{2}=0$, we find that $\phi$ satisfies the mKdV system

$$
\phi_{t}+\phi_{s s s}+\frac{3}{2}|\phi|^{2} \phi_{s}=0
$$

In the following, we shall consider the case $U=W=0$. Denote $\theta(s, t)=\int^{s} \tau\left(s^{\prime}, t\right) \mathrm{d} s^{\prime}$, $g=V \eta$. It follows from (9) that $\phi$ satisfies the equation

$$
i \phi_{t}+g_{s s}+|\phi|^{2} g-\phi \int^{s} g(\cos \theta-i \sin \theta) k_{s^{\prime}} \mathrm{d} s^{\prime}=0 .
$$

Set $\tilde{u}=\kappa \cos \theta, \tilde{v}=\kappa \sin \theta, g=g_{1}+i g_{2}$, then the equation (11) is separated to two equations

$$
\begin{aligned}
& \tilde{u}_{t}=-g_{2, s s}-\tilde{v} \partial_{s}^{-1}\left[\kappa\left(g_{1} \cos \theta+g_{2} \sin \theta\right)_{s}\right], \\
& \tilde{v}_{t}=g_{1, s s}+\tilde{u} \partial_{s}^{-1}\left[\kappa\left(g_{1} \cos \theta+g_{2} \sin \theta\right)_{s}\right] .
\end{aligned}
$$

Furthermore, letting $\tilde{u}=u+v_{s}, \tilde{v}=v-u_{s}$, and choosing the binormal velocity $V$ to be $V=\partial_{s}^{-1}\left[\left(u^{2}+v^{2}\right)_{s} / \kappa\right]$, we find that $u$ and $v$ satisfy the system

$$
\begin{aligned}
& \left(u+v_{s}\right)_{t}=-g_{2, s s}-\left(v-u_{s}\right)\left(u^{2}+v^{2}\right) \\
& \left(v-u_{s}\right)_{t}=g_{1, s s}+\left(u^{2}+v^{2}\right)\left(u+v_{s}\right)
\end{aligned}
$$


with

$$
g_{1}=\frac{\left(u+v_{s}\right)\left(u^{2}+v^{2}\right)_{s}}{\left(u+v_{s}\right)^{2}+\left(v-u_{s}\right)^{2}}, \quad g_{2}=\frac{\left(v-u_{s}\right)\left(u^{2}+v^{2}\right)_{s}}{\left(u+v_{s}\right)^{2}+\left(v-u_{s}\right)^{2}} .
$$

Applying the scaling transformations

$$
s \longmapsto s, \quad t \longmapsto \epsilon^{2} t, \quad u \longmapsto \epsilon^{-1} u, \quad v \longmapsto \epsilon^{-1} v
$$

to system (12) produces

$$
\begin{aligned}
& \left(u+v_{s}\right)_{t}=-\epsilon^{2} g_{2, s s}-\left(v-u_{s}\right)\left(u^{2}+v^{2}\right), \\
& \left(v-u_{s}\right)_{t}=\epsilon^{2} g_{1, s s}+\left(u^{2}+v^{2}\right)\left(u+v_{s}\right) .
\end{aligned}
$$

Expanding $u$ and $v$ in powers of the small parameter $\epsilon$

$$
\begin{aligned}
& u(t, s)=u_{0}(t, s)+\epsilon u_{1}(t, s)+\epsilon^{2} u_{2}(t, s)+\cdots, \\
& v(t, s)=v_{0}(t, s)+\epsilon v_{1}(t, s)+\epsilon^{2} v_{2}(t, s)+\cdots,
\end{aligned}
$$

and plugging them into system (13), we find that the leading order terms $u_{0}(t, s)$ and $v_{0}(t, s)$ satisfy the system

$$
\begin{aligned}
& \left(u_{0}+v_{0, s}\right)_{t}+\left(u_{0}^{2}+v_{0}^{2}\right)\left(v_{0}-u_{0, s}\right)=0 \\
& \left(v_{0}-u_{0, s}\right)_{t}-\left(u_{0}^{2}+v_{0}^{2}\right)\left(u_{0}+v_{0, s}\right)=0
\end{aligned}
$$

Again we use the notation $\phi(t, s)=u_{0}(t, s)+i v_{0}(t, s)$, then it is inferred from (14) that $\phi(t, s)$ satisfies the equation

$$
i\left(\phi_{t}-i \phi_{t s}\right)+|\phi|^{2}\left(\phi-i \phi_{s}\right)=0
$$

which is a dual version of the Schrödinger equation (10), and can be obtained by the approach of tri-Hamiltonian duality $[18,46]$. Equation (15) is formally completely integrable since it admits bi-Hamiltonian structure [46]

$$
\rho_{t}=\mathcal{E}_{1} \frac{\delta H_{2}}{\delta \rho}=\mathcal{E}_{2} \frac{\delta H_{1}}{\delta \rho}
$$

where $\rho=\phi-i \phi_{s}, \mathcal{E}_{1}$ and $\mathcal{E}_{2}$ defined by

$$
\mathcal{E}_{1}=\partial_{s}+i \quad \text { and } \quad \mathcal{E}_{2}(F)=\rho \partial_{s}^{-1}(\bar{\rho} F-\rho \bar{F})
$$

are compatible Hamiltonian operators, while

$$
H_{1}=\int_{\mathbb{R}}\left(|\phi|^{2}-i \bar{\phi} \phi_{s}\right) \mathrm{d} s=\int_{\mathbb{R}} \rho \bar{\phi} \mathrm{d} s, \quad H_{2}=\frac{1}{2} \int_{\mathbb{R}}|\phi|^{2}\left(|\phi|^{2}-i \bar{\phi} \phi_{s}\right) \mathrm{d} s .
$$

are the corresponding Hamiltonian functionals.

\section{Möbius sphere $\mathrm{PO}(3,1) / H$ and unit sphere $\operatorname{SO}(n+1) / \operatorname{SO}(n)$}

In this section, we give a brief account of Möbius 2-sphere $\mathrm{PO}(3,1) / H$ and unit sphere $S^{n}(1)=$ $\mathrm{SO}(n+1) / \mathrm{SO}(n)$. Please refer to the book [53] for the details of the two geometries. 


\subsection{Möbius 2-sphere}

Let $\left(u_{0}, u_{1}, u_{2}, u_{3}\right) \in \mathbb{R}^{4}$, we define the inner product on $\mathbb{R}^{4}$ by

$$
\langle x, y\rangle=x^{T} \Lambda_{3,1} y
$$

where $x, y \in \mathbb{R}^{4}$, and the matrix $\Lambda_{3,1}$ is

$$
\Lambda_{3,1}=\left(\begin{array}{cccc}
0 & 0 & 0 & -1 \\
0 & 1 & 0 & 0 \\
0 & 0 & 1 & 0 \\
-1 & 0 & 0 & 0
\end{array}\right)
$$

A vector field $x \in \mathbb{R}^{4}$ is said to be light-like, if it satisfies $\langle x, x\rangle=0$. All the light-like vector fields form a set $L$, which is called optical cone, defined by the equation

$$
2 x_{0} x_{3}-x_{1}^{2}-x_{2}^{2}=0 .
$$

Clearly it is homogeneous, namely for any $\lambda \in \mathbb{R}$, if $x \in L$, then $\lambda x \in L$. The projectivisation of $L$ is said to be Möbius 2-sphere, which is isomorphic to $\mathbb{S}^{2}$. Recall that

$$
\mathrm{O}(3,1)=\left\{g \in \mathrm{GL}(4, \mathbb{R}): g^{T} \Lambda_{3,1} g=\Lambda_{3,1}\right\},
$$

and the Möbius group is defined to be $\mathrm{PO}(3,1)=\mathrm{O}(3,1) / \pm I$. We denote

$$
\begin{aligned}
H & =\left\{h \in \mathrm{PO}(3,1): h\left[e_{3}\right]=\left[e_{3}\right]\right\} \\
& =\left\{\left(\begin{array}{ccc}
a^{-1} & 0 & 0 \\
v & A & 0 \\
b & \eta^{T} & a
\end{array}\right) \in \mathrm{O}(3,1) ; A \in \mathrm{O}(2), a \in \mathbb{R}^{+}, v \in \mathbb{R}^{2}\right\},
\end{aligned}
$$

where $\left[e_{3}\right]$ denotes the equivalent class of $e_{3}$ in $P\left(\mathbb{R}^{4}\right),\left[e_{3}\right]=(0,0,0, *)$. A straightforward computation gives

$$
\eta=a A^{T} v, \quad b=\frac{a}{2} v^{T} v=\frac{a}{2}\|v\|^{2} .
$$

It is easy to verify that the group $\mathrm{PO}(3,1)$ acts on the Möbius sphere transitively (the group action is the usual conformal transformation).

Definition 1. The Klein pair $(\mathrm{PO}(3,1), H)$ is called the Möbius 2-sphere.

For any $g \in \mathrm{PO}(3,1)$, there exists a unique decomposition around the identity of the group

$$
g=g_{1} g_{0} g_{-1}=\left(\begin{array}{ccc}
1 & 0 & 0 \\
v & I_{2} & 0 \\
\frac{1}{2}\|v\|^{2} & v^{T} & 1
\end{array}\right)\left(\begin{array}{ccc}
a^{-1} & 0 & 0 \\
0 & A & 0 \\
0 & 0 & a
\end{array}\right)\left(\begin{array}{ccc}
1 & u^{T} & \frac{1}{2}\|u\|^{2} \\
0 & I_{2} & u \\
0 & 0 & 1
\end{array}\right)
$$

where $a \in \mathbb{R}^{+}, A \in \mathrm{O}(2), u, v \in \mathbb{R}^{2}, h=g_{1} g_{0} \in H, u$ in $g_{-1}$ part represent a local coordinate of the point in $\mathrm{PO}(3,1) / H$. For the corresponding Lie algebra $\mathfrak{g}$, there exists also a decomposition

$$
\mathfrak{o}(3,1)=\mathfrak{g}=\mathfrak{g}_{1} \oplus \mathfrak{g}_{0} \oplus \mathfrak{g}_{-1},
$$

where

$$
\left(\begin{array}{ccc}
0 & 0 & 0 \\
p & 0 & 0 \\
0 & p^{T} & 0
\end{array}\right) \in \mathfrak{g}_{1}, \quad\left(\begin{array}{ccc}
-\epsilon & 0 & 0 \\
0 & S & 0 \\
0 & 0 & \epsilon
\end{array}\right) \in \mathfrak{g}_{0}, \quad\left(\begin{array}{ccc}
0 & q^{T} & 0 \\
0 & 0 & q \\
0 & 0 & 0
\end{array}\right) \in \mathfrak{g}_{-1},
$$

$p, q \in \mathbb{R}^{2}, \epsilon \in \mathbb{R}, S \in \mathrm{O}(2)$. The Lie algebra of the isotropy group $H$ is $\mathfrak{h}=\mathfrak{g}_{1} \oplus \mathfrak{g}_{0}$ while $\mathfrak{g} / \mathfrak{h}=\mathfrak{g}_{-1}$ is identified to the tangent space of the conformal sphere $\mathrm{PO}(3,1) / H$. 


\section{$3.2 n$-dimensional sphere $\mathbb{S}^{n}(1)=\mathrm{SO}(n+1) / \mathrm{SO}(n)$}

The $n$-dimensional unit-sphere is also a homogeneous space $M=G / H=\mathrm{SO}(n+1) / \mathrm{SO}(n)$. The corresponding Lie algebra has the following Cartan-Killing decomposition

$$
\mathfrak{s o}(n+1)=\mathfrak{h} \oplus \mathfrak{m}=\mathfrak{s o}(n) \oplus \mathbb{R}^{n},
$$

with

$$
\left(\begin{array}{cc}
0 & -p^{T} \\
p & 0
\end{array}\right) \in \mathfrak{m}, \quad\left(\begin{array}{cc}
0 & 0 \\
0 & \Theta
\end{array}\right) \in \mathfrak{h},
$$

where $p \in \mathbb{R}^{n}, \Theta \in \mathfrak{s o}(n)$, and the decomposition satisfies

$$
[\mathfrak{h}, \mathfrak{h}] \subset \mathfrak{h}, \quad[\mathfrak{h}, \mathfrak{m}] \subset \mathfrak{m}, \quad[\mathfrak{m}, \mathfrak{m}] \subset \mathfrak{h},
$$

where $\mathfrak{m}$ is identified to the tangent space $T_{x} M \cong \mathbb{R}^{n}$ of $M=\mathrm{SO}(n+1) / \mathrm{SO}(n)$. The flat Cartan connection of principle $\mathrm{SO}(n)$ bundle $\mathrm{SO}(n+1) \rightarrow \mathbb{S}^{n}$ is given by the Maurer-Cartan form of Lie group $\mathrm{SO}(n+1)$. The Cartan structure equation reads as

$$
\Omega=\mathrm{d} \omega+\frac{1}{2}[\omega, \omega]=0,
$$

where $\mathfrak{s o}(n+1)$-valued one-form $\omega$ is decomposed to

$$
\omega=\omega_{H}+\theta, \quad \omega_{H} \in \Lambda^{1}(P, \mathfrak{h}), \quad \theta \in \Lambda^{1}(P, \mathfrak{g} / \mathfrak{h}),
$$

where $\mathfrak{g} / \mathfrak{h}$-valued $\theta$ represents a linear coframe, $\mathfrak{h}$-valued $\omega_{H}$ represents a linear connection on $\mathbb{S}^{n}$. The corresponding Cartan structure equation is separated to

$$
\mathfrak{J} \equiv \mathrm{d} \theta+\frac{1}{2}\left[\omega_{H}, \theta\right]+\frac{1}{2}\left[\theta, \omega_{H}\right]=0
$$

and

$$
\mathfrak{R} \equiv \mathrm{d} \omega_{H}+\frac{1}{2}\left[\omega_{H}, \omega_{H}\right]=-\frac{1}{2}[\theta, \theta],
$$

where $\mathfrak{J}$ and $\mathfrak{R}$ are called torsion and curvature forms, respectively.

\section{Curve flows in $\mathrm{PO}(3,1) / H$ and the complex $\mathrm{CH}$ equation}

For the Möbius geometry $\mathrm{PO}(3,1) / H$, its Cartan connection takes values on $\mathfrak{g}=o(3,1)$, with the form

$$
\omega=\left(\begin{array}{ccc}
-\epsilon & \xi^{T} & 0 \\
\eta & \Theta & \xi \\
0 & \eta^{T} & \epsilon
\end{array}\right)
$$

and the Cartan structure equation reads as

$$
\Omega=\mathrm{d} \omega+\frac{1}{2}[\omega, \omega]=0 .
$$

Let's consider the invariant curve flows for curves $\gamma(x, t)=\left(u_{1}(x, t), u_{2}(x, t)\right)$ on the conformal sphere $M^{2}=\mathrm{PO}(3,1) / H$, where $x$ denotes the parameter of the curves, $t$ is the time variable, $u_{1}$ and $u_{2}$ denote the local coordinates on $M^{2}$. Let $\gamma_{t}=\gamma_{*} \frac{\partial}{\partial t}$ be the evolutionary vector field of 
the curves, $\gamma_{x}=\gamma_{*} \frac{\partial}{\partial x}$ denotes the tangent vector of the curves. Assuming that the curve flow is intrinsic, namely the parameter $x$ for the curve does not depend on time $t$, we have

$$
\left[\gamma_{x}, \gamma_{t}\right]=0
$$

It was shown in $[16,36]$ that there exists a conformally equivariant moving frame (the Frenet frame) $\rho=\rho(x, t) \in \mathrm{PO}(3,1)$ along the curve $\gamma(x, t) \subset M^{2}$. Let $D_{x}$ and $D_{t}$ denote respectively the vector field $\frac{d}{d x}$ and $\frac{d}{d t}$ along the curves $\rho$ in $\mathrm{PO}(3,1)$, then the Frenet formulae for the conformally parametric curves is

$$
\rho_{x}=\rho \hat{\omega}\left(D_{x}\right)
$$

with

$$
\hat{\omega}\left(D_{x}\right)=\left(\begin{array}{cccc}
0 & 1 & 0 & 0 \\
k_{1} & 0 & 0 & 1 \\
k_{2} & 0 & 0 & 0 \\
0 & k_{1} & k_{2} & 0
\end{array}\right),
$$

where $k_{1}$ and $k_{2}$ are the conformally differential invariants for the curve $\gamma(x, t)$. The time evolution for the frame $\rho(x, t)$ can be written as

$$
\rho_{t}=\rho \hat{\omega}\left(D_{t}\right)
$$

where

$$
\hat{\omega}\left(D_{t}\right)=\left(\begin{array}{cccc}
-\epsilon & h_{1} & h_{2} & 0 \\
f_{1} & 0 & -\alpha & h_{1} \\
f_{2} & \alpha & 0 & h_{2} \\
0 & f_{1} & f_{2} & \epsilon
\end{array}\right),
$$

and $\epsilon, \alpha, f_{i}, h_{i}(i=1,2)$ are some conformal differential invariants related to $k_{1}$ and $k_{2}$, to be determined. By the Cartan structure equation, one gets

$$
\Omega\left(D_{x}, D_{t}\right)=\frac{d}{d t} \hat{\omega}\left(D_{x}\right)-\frac{d}{d x} \hat{\omega}\left(D_{t}\right)-\left[\hat{\omega}\left(D_{x}\right), \hat{\omega}\left(D_{t}\right)\right]=0 .
$$

Plugging (16) and (17) into (18) results in the following equations

$$
\begin{aligned}
& \epsilon=-h_{1, x}, \quad \alpha=h_{2, x}, \\
& f_{1}=\epsilon_{x}+k_{1} h_{1}+k_{2} h_{2}, \\
& f_{2}=\alpha_{x}+k_{2} h_{1}-k_{1} h_{2}, \\
& k_{1, t}=f_{1, x}-k_{1} \epsilon+\alpha k_{2}, \\
& k_{2, t}=f_{2, x}-\epsilon k_{2}-\alpha k_{1},
\end{aligned}
$$

where (19) is the torsion part (i.e.,the $\mathfrak{g}_{-1}$ part) of the Cartan structure equation (18), which can be written as

$$
\left(\begin{array}{c}
\epsilon \\
\alpha
\end{array}\right)=\left(\begin{array}{c}
-\partial_{x} \\
\partial_{x}
\end{array}\right)\left(\begin{array}{l}
h_{1} \\
h_{2}
\end{array}\right) \equiv J_{1}\left(\begin{array}{l}
h_{1} \\
h_{2}
\end{array}\right) .
$$

Inserting (19) into (20) and (21) gives

$$
f_{1}=-h_{1, x x}+k_{1} h_{1}+k_{2} h_{2}, \quad f_{2}=h_{2, x x}+k_{2} h_{1}-k_{1} h_{2} .
$$


Substituting (19) and (24) into (22) and (23), we obtain the evolution equations for the curvatures $k_{1}$ and $k_{2}[32,36]$

$$
\begin{aligned}
& k_{1, t}=-h_{1, x x x}+2 k_{1} h_{1, x}+k_{1, x} h_{1}+2 k_{2} h_{2, x}+k_{2, x} h_{2}, \\
& k_{2, t}=h_{2, x x x}-2 k_{1} h_{2, x}-k_{1, x} h_{2}+2 k_{2} h_{1, x}+k_{2, x} h_{1},
\end{aligned}
$$

which is equivalent to

$$
\left(\begin{array}{l}
k_{1} \\
k_{2}
\end{array}\right)_{t}=\left(\begin{array}{cc}
-\partial^{3}+k_{1} \partial+\partial k_{1} & k_{2} \partial+\partial k_{2} \\
k_{2} \partial+\partial k_{2} & \partial^{3}-k_{1} \partial-\partial k_{1}
\end{array}\right)\left(\begin{array}{l}
h_{1} \\
h_{2}
\end{array}\right) \equiv J_{2}\left(\begin{array}{l}
h_{1} \\
h_{2}
\end{array}\right) .
$$

The following cases are considered.

Case 1. Setting $k_{1}=1 / 2+m \equiv 1 / 2+u-u_{x x}, k_{2}=n \equiv v-v_{x x}, h_{1}=1-u$ and $h_{2}=v$ in (25), we obtain the new two-component $\mathrm{CH}$ equation

$$
\begin{aligned}
& m_{t}+2 u_{x} m+u m_{x}-2 v_{x} n-v n_{x}=0, \\
& n_{t}+2 u_{x} n+u n_{x}+2 v_{x} m+v m_{x}=0 .
\end{aligned}
$$

The above system admits the following Lax-pair

$$
\phi_{x}=U \phi, \quad \phi_{t}=V \phi,
$$

with

$$
U=\left(\begin{array}{cccc}
0 & \frac{1}{2}+\lambda m & \lambda n & 0 \\
1 & 0 & 0 & \frac{1}{2}+\lambda m \\
0 & 0 & 0 & \lambda n \\
0 & 1 & 0 & 0
\end{array}\right) \quad \text { and } \quad V=\left(\begin{array}{cccc}
-u_{x} & f_{1} & f_{2} & 0 \\
\lambda^{-1}-u & 0 & v_{x} & f_{1} \\
v & -v_{x} & 0 & f_{2} \\
0 & \lambda^{-1}-u & v & u_{x}
\end{array}\right)
$$

where $f_{1}=\frac{1}{2}\left(\lambda^{-1}+u\right)+\lambda(v n-u m), f_{2}=\frac{1}{2} v-\lambda(u n+v m)$.

Case 2. Setting $k_{1}=m=-u_{x x}, k_{2}=n=-v_{x x}, h_{1}=1-u, h_{2}=v$, we arrive at the complex Hunter-Saxton equation

$$
\begin{aligned}
& m_{t}+2 u_{x} m+u m_{x}-2 v_{x} n-v n_{x}=0, \\
& n_{t}+2 u_{x} n+u n_{x}+2 v_{x} m+v m_{x}=0,
\end{aligned}
$$

which admits the Lax-pair (27) with

$$
U=\left(\begin{array}{cccc}
0 & -\lambda u_{x x} & -\lambda v_{x x} & 0 \\
1 & 0 & 0 & -\lambda u_{x x} \\
0 & 0 & 0 & -\lambda v_{x x} \\
0 & 1 & 0 & 0
\end{array}\right)
$$

and

$$
V=\left(\begin{array}{cccc}
-u_{x} & \lambda(v n-u m) & -\lambda(u n+v m) & 0 \\
\lambda^{-1}-u & 0 & v_{x} & \lambda(v n-u m) \\
v & -v_{x} & 0 & -\lambda(u n+v m) \\
0 & \lambda^{-1}-u & v & u_{x}
\end{array}\right) .
$$

Case 3. In (25), letting $h_{1}$ and $h_{2}$ satisfy

$$
k_{1}=\frac{1}{2} \frac{h_{1}^{2}-h_{2}^{2}}{\left(h_{1}^{2}+h_{2}^{2}\right)^{2}}, \quad k_{2}=-\frac{h_{1} h_{2}}{\left(h_{1}^{2}+h_{2}^{2}\right)^{2}},
$$

we get the two-component Harry-Dym equation

$$
\begin{aligned}
& h_{1, t}=\left(h_{1}^{3}-3 h_{1} h_{2}^{2}\right) h_{1, x x x}+\left(h_{2}^{3}-3 h_{1}^{2} h_{2}\right) h_{2, x x x}, \\
& h_{2, t}=\left(h_{2}^{3}-3 h_{1}^{2} h_{2}\right) h_{1, x x x}-\left(h_{1}^{3}-3 h_{1} h_{2}^{2}\right) h_{2, x x x} .
\end{aligned}
$$


Remark 1. Marí Beffa $[32,36]$ showed that the complex KdV equation arises from the invariant curve motion in Möbius 2-sphere. Indeed, taking $h_{1}=-k_{1}, h_{2}=-k_{2}$ in (25) yields the complex $\mathrm{KdV}$ equation

$$
k_{1, t}=k_{1, x x x}-3 k_{1} k_{1, x}+3 k_{2} k_{2, x}, \quad k_{2, t}=k_{2, x x x}-3 k_{1} k_{2, x}-3 k_{2} k_{1, x} .
$$

Its Hamiltonian structure $J_{2}$, see (25), was originally derived in [32]. One can see that the biHamiltonian structure $J_{1}$ and $J_{2}$ of the complex $\mathrm{KdV}$ equation comes from the Cartan structure equation for the conformal invariant curve flow. According to the decomposition of the Lie algebra

$$
\mathfrak{g}=\mathfrak{g}_{1} \oplus \mathfrak{g}_{0} \oplus \mathfrak{g}_{-1},
$$

the Cartan curvature form $\Omega$ is decomposed to $\Omega=\Omega_{1}+\Omega_{0}+\Omega_{-1}$, where $J_{1}$ comes from torsion part of the structure equation, i.e., the $\Omega_{-1}$ part, and $J_{2}$ arises from the $\Omega_{1}$ part. In the sequel, we will show that the complex $\mathrm{CH}$ equation admits a bi-Hamiltonian structure $\hat{\mathcal{J}}_{1}$ and $\hat{\mathcal{J}}_{2}$. It turns out that the complex $\mathrm{CH}$ equation is a dual version of the complex $\mathrm{KdV}$ equation (in the sense of [46]).

It is well-known that the $\mathrm{CH}$ equation is a bi-Hamiltonian system [8]

$$
m_{t}=\mathcal{J} \frac{\delta H_{1}}{\delta m}=\mathcal{D} \frac{\delta H_{2}}{\delta m}
$$

where $\mathcal{J}=-(m \partial+\partial m)$ and $\mathcal{D}=-\left(\partial-\partial^{3}\right)$ are Hamiltonian operators, $H_{1}=-\frac{1}{2} \int u m \mathrm{~d} x$ and $H_{2}=-\frac{1}{2} \int u\left(u^{2}+u_{x}^{2}\right) \mathrm{d} x$ are the corresponding Hamiltonian functionals. As for the CH equation, the complex CH equation can be obtained by the approach of tri-Hamiltonian duality [46]. Indeed, we have the following result.

Theorem 1. The complex $C H$ equation (26) is a bi-Hamiltonian system, which can be written as

$$
\left(\begin{array}{c}
m \\
n
\end{array}\right)_{t}=\hat{\mathcal{J}}_{1}\left(\begin{array}{l}
\frac{\delta \hat{H}_{2}}{\delta m} \\
\frac{\delta \hat{H}_{2}}{\delta n}
\end{array}\right)=\hat{\mathcal{J}}_{2}\left(\begin{array}{l}
\frac{\delta \hat{H}_{1}}{\delta m} \\
\frac{\delta \hat{H}_{1}}{\delta n}
\end{array}\right)
$$

with

$$
\hat{\mathcal{J}}_{1}=\left(\begin{array}{cc}
\partial^{3}-\partial & 0 \\
0 & \partial-\partial^{3}
\end{array}\right), \quad \hat{\mathcal{J}}_{2}=\left(\begin{array}{cc}
m \partial+\partial m & n \partial+\partial n \\
n \partial+\partial n & -m \partial-\partial m
\end{array}\right)
$$

and

$$
\hat{H}_{1}=\frac{1}{2} \int(v n-u m) \mathrm{d} x, \quad \hat{H}_{2}=\frac{1}{2} \int\left[u\left(u^{2}+u_{x}^{2}\right)-u\left(3 v^{2}+v_{x}^{2}\right)-2 v u_{x} v_{x}\right] \mathrm{d} x .
$$

Proof. Clearly, $\hat{\mathcal{J}}_{1}$ and $\hat{\mathcal{J}}_{2}$ are skew symmetric. To prove they are Hamiltonian operators, it suffices to prove that the Poisson bracket defined by $\hat{\mathcal{J}}_{2}$ satisfies the Jacobi identity.

The bi-vector associated with $\hat{\mathcal{J}}_{2}$ is defined by [44]

$$
\Theta_{\hat{\mathcal{J}}_{2}}=\frac{1}{2} \int_{\mathbb{R}}\left(\vartheta \wedge \hat{\mathcal{J}}_{2} \vartheta\right) \mathrm{d} x=\int_{\mathbb{R}}\left(m \theta \wedge \theta_{x}+n \theta \wedge \zeta_{x}+n \zeta \wedge \theta_{x}-m \zeta \wedge \zeta_{x}\right),
$$

where $\vartheta=(\theta, \zeta), \theta$ and $\zeta$ denote the basic unit vectors corresponding to $m$ and $n$, respectively, the notation $\wedge$ denotes the usual inner product between $\vartheta$ and $\hat{\mathcal{J}}_{2} \vartheta$. It suffices to show that the Schouten bracket vanishes, namely $\left[\hat{\mathcal{J}}_{2}, \hat{\mathcal{J}}_{2}\right]=0$. In terms of

$$
\operatorname{pr} v_{\hat{\mathcal{J}}_{2} \vartheta}(m)=2 m \theta_{x}+m_{x} \theta+2 n \zeta_{x}+n_{x} \zeta, \quad \operatorname{pr} v_{\hat{\mathcal{J}}_{2} \vartheta}(n)=2 n \theta_{x}+n_{x} \theta-2 m \zeta_{x}-m_{x} \zeta,
$$


a direct computation shows

$$
\begin{aligned}
{\left[\hat{\mathcal{J}}_{1}, \hat{\mathcal{J}}_{2}\right]=} & \operatorname{pr} v_{\hat{\mathcal{J}}_{2} v}\left(\Theta_{\hat{\mathcal{J}}_{2}}\right) \\
= & \int\left(2 n \zeta_{x} \wedge \theta \wedge \theta_{x}+n_{x} \zeta \wedge \theta \wedge \theta_{x}+2 n \theta_{x} \wedge \theta \wedge \zeta_{x}-m_{x} \zeta \wedge \theta \wedge \zeta_{x}\right. \\
& \left.+n_{x} \theta \wedge \zeta \wedge \theta_{x}-2 m \zeta_{x} \wedge \zeta \wedge \theta_{x}-2 m \theta_{x} \wedge \zeta \wedge \zeta_{x}-m_{x} \theta \wedge \zeta \wedge \theta_{x}\right) \mathrm{d} x=0
\end{aligned}
$$

where the skew-symmetric property for the wedge product is used.

Next, we prove that the Hamiltonian operators $\hat{\mathcal{J}}_{1}$ and $\hat{\mathcal{J}}_{2}$ are compatible, i.e.

$$
\left[\hat{\mathcal{J}}_{1}, \hat{\mathcal{J}}_{2}\right]+\left[\hat{\mathcal{J}}_{2}, \hat{\mathcal{J}}_{1}\right]=\operatorname{pr} v_{\hat{\mathcal{J}}_{1} \vartheta}\left(\Theta_{\hat{\mathcal{J}}_{2}}\right)+\operatorname{pr} v_{\hat{\mathcal{J}}_{2} \vartheta}\left(\Theta_{\hat{\mathcal{J}}_{1}}\right)=0
$$

Note that

$$
\operatorname{pr} v_{\hat{\mathcal{J}}_{2} \vartheta}\left(\Theta_{\hat{\mathcal{J}}_{1}}\right)=0, \quad \operatorname{pr} v_{\hat{\mathcal{J}}_{1} \vartheta}(m)=\theta_{x x x}-\theta_{x}, \quad \operatorname{pr} v_{\hat{\mathcal{J}}_{1} \vartheta}(n)=\zeta_{x}-\zeta_{x x x} .
$$

Through integration by parts, we get

$$
\begin{aligned}
{\left[\hat{\mathcal{J}}_{1}, \hat{\mathcal{J}}_{2}\right]+\left[\hat{\mathcal{J}}_{2}, \hat{\mathcal{J}}_{1}\right]=} & \operatorname{pr} v_{\hat{\mathcal{J}}_{1} \vartheta}\left(\Theta_{\hat{\mathcal{J}}_{2}}\right)+\operatorname{pr} v_{\hat{\mathcal{J}}_{2} \vartheta}\left(\Theta_{\hat{\mathcal{J}}_{1}}\right) \\
= & \int\left(\theta_{x x x} \wedge \theta \wedge \theta_{x}-\zeta_{x x x} \wedge \theta \wedge \zeta_{x}+\zeta_{x} \wedge \zeta \wedge \theta_{x}-\zeta_{x x x} \wedge \zeta \wedge \theta_{x}\right. \\
& \left.-\theta_{x x x} \wedge \zeta \wedge \zeta_{x}+\theta_{x} \wedge \zeta \wedge \zeta_{x}\right) \mathrm{d} x \\
= & \int\left(-\zeta_{x x x} \wedge \theta \wedge \zeta_{x}-\zeta_{x x x} \wedge \zeta \wedge \theta_{x}-\theta_{x x x} \wedge \zeta \wedge \zeta_{x}\right) \mathrm{d} x \\
= & \int\left(-\zeta_{x x x} \wedge \theta \wedge \zeta_{x}-\zeta_{x x x} \wedge \zeta \wedge \theta_{x}-\theta_{x} \wedge \zeta_{x} \wedge \zeta_{x x}-\theta_{x} \wedge \zeta \wedge \zeta_{x x x}\right) \mathrm{d} x \\
= & \int\left(-\zeta_{x x x} \wedge \theta \wedge \zeta_{x}-\theta_{x} \wedge \zeta_{x} \wedge \zeta_{x x}\right) \mathrm{d} x=0 .
\end{aligned}
$$

Thus $\hat{\mathcal{J}}_{1}$ and $\hat{\mathcal{J}}_{2}$ are a Hamiltonian-pair. Let's write the complex $\mathrm{CH}$ equation (26) as follows

$$
\left(\begin{array}{c}
m \\
n
\end{array}\right)_{t}=\hat{\mathcal{J}}_{2}\left(\begin{array}{c}
\frac{\delta \hat{H}_{1}}{\delta m} \\
\frac{\delta \hat{H}_{1}}{\delta n}
\end{array}\right) .
$$

It is easy to get

$$
\frac{\delta \hat{H}_{1}}{\delta m}=-u, \quad \frac{\delta \hat{H}_{1}}{\delta n}=v .
$$

Thus

$$
\frac{\delta \hat{H}_{1}}{\delta u}=-m, \quad \frac{\delta \hat{H}_{1}}{\delta v}=n .
$$

Hence we deduce that

$$
\hat{H}_{1}=\frac{1}{2} \int(v n-u m) \mathrm{d} x
$$

To compute $\hat{H}_{2}$, we write $(26)$ as

$$
\begin{aligned}
& m_{t}=-\partial\left(\frac{3}{2} u^{2}-\frac{1}{2} u_{x}^{2}-u u_{x x}-\frac{3}{2} v^{2}+\frac{1}{2} v_{x}^{2}+v v_{x x}\right), \\
& n_{t}=\partial\left(u v_{x x}+v u_{x x}+u_{x} v_{x}-3 u v\right) .
\end{aligned}
$$


Since $\hat{H}_{2}$ satisfies

$$
m_{t}=-\partial\left(1-\partial^{2}\right) \frac{\delta \hat{H}_{2}}{\delta m}=-\partial \frac{\delta \hat{H}_{2}}{\delta u}, \quad n_{t}=\partial\left(1-\partial^{2}\right) \frac{\delta \hat{H}_{2}}{\delta n}=\partial \frac{\delta \hat{H}_{2}}{\delta v} .
$$

It follows that

$$
\begin{aligned}
\frac{\delta \hat{H}_{2}}{\delta u} & =\frac{3}{2} u^{2}-\frac{1}{2} u_{x}^{2}-u u_{x x}-\frac{3}{2} v^{2}+\frac{1}{2} v_{x}^{2}+v v_{x x}, \\
\frac{\delta \hat{H}_{2}}{\delta v} & =u v_{x x}+v u_{x x}+u_{x} v_{x}-3 u v .
\end{aligned}
$$

Note that

$$
\begin{aligned}
& \frac{3}{2} u^{2}-\frac{1}{2} u_{x}^{2}-u u_{x x}=\frac{\delta}{\delta u} \frac{1}{2} \int\left(u^{3}+u u_{x}^{2}\right) \mathrm{d} x, \\
& \frac{\delta}{\delta u} \int u v^{2} \mathrm{~d} x=v^{2} \\
& \frac{\delta}{\delta v} \int u v^{2} \mathrm{~d} x=2 u v \\
& \frac{\delta}{\delta u} \int\left(-\frac{1}{2} u v_{x}^{2}-u_{x} v v_{x}\right) \mathrm{d} x=\frac{1}{2} v_{x}^{2}+v v_{x x} .
\end{aligned}
$$

Hence we arrive at

$$
\hat{H}_{2}=\frac{1}{2} \int\left(u^{3}+u u_{x}^{2}-3 u v^{2}-u v_{x}^{2}-2 u_{x} v v_{x}\right) \mathrm{d} x .
$$

\section{Curve flows on $\mathbb{S}^{n}(1)$ and multi-component modified $\mathrm{CH}$ equations}

Assume that $\gamma(x, t)$ is a curve flow on unit sphere $\mathbb{S}^{n}(1)=\mathrm{SO}(n+1) / \mathrm{SO}(n)$, which satisfies $\|\gamma\|=1$, where $x$ is the invariant arc-length parameter, $t$ is the time. The natural frame of the curve $\gamma \in \mathbb{S}^{n}(1)$ is $\left\{e_{1}=\gamma_{x}, e_{2}, \ldots, e_{n}\right\}$. Let $\rho=\left(e_{0}=\gamma, e_{1}, \ldots, e_{n}\right) \in \mathrm{SO}(n+1)$ be the lift from $\mathbb{S}^{n}(1)$ to bundle space $\mathrm{SO}(n+1)$, and $D_{x}$ and $D_{t}$ denote respectively the tangent and evolutionary vector field. It follows that

$$
\rho_{x}=\rho \hat{\omega}\left(D_{x}\right)
$$

where $\hat{\omega}$ is the Cartan connection

$$
\hat{\omega}\left(D_{x}\right)=\left(\begin{array}{ccc}
0 & -1 & \overrightarrow{0}^{T} \\
1 & 0 & -\vec{k}^{T} \\
\overrightarrow{0} & \vec{k} & O
\end{array}\right), \quad O \in \mathfrak{s o}(n-1),
$$

(the natural frame formulae (28) for curves on the sphere comes out from the Frenet formulae [15] through the Hasimoto transformation). Here $\vec{k}=\left(k_{1}, k_{2}, \ldots, k_{n-1}\right)$ is the natural curvature vector of $\gamma$.

Assume that the curve flow is governed by

$$
\gamma_{t}=f e_{1}+h_{1} e_{2}+h_{2} e_{3}+\cdots+h_{n-1} e_{n}
$$

where the tangent velocity $f$ and normal velocities $h_{i}(i=1,2, \ldots, n-1)$ depend on the curvatures and their derivatives with respect to arc-length $x$. 
The induced time evolution for the frame is

$$
\rho_{t}=\rho \hat{\omega}\left(D_{t}\right),
$$

with

$$
\hat{\omega}\left(D_{t}\right)=\left(\begin{array}{ccc}
0 & -f & -\vec{h}^{T} \\
f & 0 & -\vec{\xi}^{T} \\
\vec{h} & \vec{\xi} & \Theta
\end{array}\right), \quad \Theta \in \mathfrak{s o}(n-1),
$$

where $\vec{h}, \vec{\xi} \in \mathbb{R}^{n-1}, \vec{\xi}$ is a unknown vector, which will be determined later by the structure equations.

First, we assume that the flow is intrinsic, namely, the distribution $\left\{D_{x}, D_{t}\right\}$ satisfies $\left[D_{x}, D_{t}\right]=0$ so that the integral submanifold is a smooth two-dimensional surface on Lie group $\mathrm{SO}(n+1)$. Making use of the Cartan structure equation

$$
\frac{d}{d t} \hat{\omega}\left(D_{x}\right)-\frac{d}{d x} \hat{\omega}\left(D_{t}\right)-\left[\hat{\omega}\left(D_{x}\right), \hat{\omega} a\left(D_{t}\right)\right]=0,
$$

one gets the following equations

$$
\begin{aligned}
& f_{x}=\langle\vec{k}, \vec{h}\rangle, \\
& \vec{\xi}=\vec{h}_{x}+f \vec{k}, \\
& \Theta_{x}=\vec{k} \otimes \vec{\xi}-\vec{\xi} \otimes \vec{k}, \\
& \vec{k}_{t}=\vec{\xi}_{x}-\Theta \vec{k}+\vec{h},
\end{aligned}
$$

where (29) is the arc-length preserving condition.

For convenience, the following notations are used. For any $\vec{a}, \vec{b} \in \mathbb{R}^{n-1},\langle\vec{a}, \vec{b}\rangle$ denotes the usual Euclidean inner product, i.e., $\langle\vec{a}, \vec{b}\rangle=\vec{a}^{T} \vec{b}, \vec{a} \otimes \vec{b}$ denotes the tensor product, namely

$$
\vec{a} \otimes \vec{b}=\left(\begin{array}{cccc}
a_{1} b_{1} & a_{1} b_{2} & \cdots & a_{1} b_{n-1} \\
a_{2} b_{1} & a_{2} b_{2} & \cdots & a_{2} b_{n-1} \\
\cdots & \cdots & \cdots & \cdots \\
a_{n-1} b_{1} & a_{n-1} b_{2} & \cdots & a_{n-1} b_{n-1}
\end{array}\right) .
$$

Define $\vec{a} \wedge \vec{b}=\vec{a} \otimes \vec{b}-\vec{b} \otimes \vec{a}$. From (29) and (30), it follows that

$$
f=\partial_{x}^{-1}\langle\vec{k}, \vec{h}\rangle, \quad \vec{\xi}=\vec{h}_{x}+\left(\partial_{x}^{-1}\langle\vec{k}, \vec{h}\rangle\right) \vec{k} .
$$

In view of (31), we have

$$
\Theta=\partial_{x}^{-1}(\vec{k} \wedge \vec{\xi})
$$

Plugging (34) and (33) into (32) leads to the equation for the curvature vector

$$
\vec{k}_{t}=\vec{h}_{x x}+\langle\vec{k}, \vec{k}\rangle \vec{h}+\left(\partial_{x}^{-1}\langle\vec{k}, \vec{h}\rangle\right) \vec{k}_{x}+\left(\partial_{x}^{-1}\left(\vec{k}_{x} \wedge \vec{h}\right)\right) \vec{k}+\vec{h}
$$

where the identity for vectors

$$
(\vec{a} \wedge \vec{b}) \cdot \vec{c}=\langle\vec{b}, \vec{c}\rangle \vec{a}-\langle\vec{a}, \vec{c}\rangle \vec{b}
$$

was used.

Analogous to the derivation for the $\mathrm{CH}$ equation [18] and the modified $\mathrm{CH}$ equation [46], we restrict our attention to the following cases. 
Case 1. $\vec{h}=\vec{u}_{x}, \vec{k}=\tilde{m}=\vec{u}+\vec{u}_{x x}$. In this case, the tangent velocity $f$ is determined by

$$
f=\partial_{x}^{-1}\left\langle\vec{u}+\vec{u}_{x x}, \vec{u}_{x}\right\rangle=\frac{1}{2}\left(|\vec{u}|^{2}+\left|\vec{u}_{x}\right|^{2}\right)+c_{0} \equiv \frac{1}{2} \tilde{Q}+c_{0}
$$

where $c_{0}$ is an integration constant. Substituting (36) with $c_{0}=-1$ together with the expressions for $\vec{k}$ and $\vec{h}$ into (35), and noting that

$$
\begin{aligned}
\left(\partial_{x}^{-1}\left(\vec{k}_{x} \wedge \vec{h}\right)\right) \vec{k} & =\left(\partial_{x}^{-1}\left(\vec{u}_{x x x} \wedge \vec{u}_{x}\right)\right) \tilde{m}=\left(\vec{u}_{x x} \wedge \vec{u}_{x}\right) \tilde{m} \\
& =-\left(\vec{u} \wedge \vec{u}_{x}\right) \tilde{m}-\left(\vec{u}_{x} \wedge \tilde{m}\right) \tilde{m}=-\left(\vec{u} \wedge \vec{u}_{x}\right) \tilde{m}-\langle\tilde{m}, \tilde{m}\rangle \vec{u}_{x}+\frac{1}{2} \tilde{Q}_{x} \tilde{m}
\end{aligned}
$$

we arrive at the multi-component modified $\mathrm{CH}$ equation

$$
\tilde{m}_{t}=\frac{1}{2}(\tilde{m} \tilde{Q})_{x}-\left(\vec{u} \wedge \vec{u}_{x}\right) \tilde{m} .
$$

Thus we have established the following result.

Theorem 2. Assume that curves $\gamma(x, t)$ on the sphere $\mathbb{S}^{n}(1)(n \geq 1)$ are governed by the flow

$$
\gamma_{t}=\left(\frac{1}{2} \tilde{Q}-1\right) e_{1}+\sum_{j=1}^{n-1} u_{j, x} e_{j+1}
$$

where $\left\{e_{1}, e_{2}, \ldots, e_{n}\right\}$ is the natural frame of the curve $\gamma(x, t),\left(u_{1}, u_{2}, \ldots, u_{n-1}\right)$ is defined by the curvatures $\vec{k}=\tilde{m}=\vec{u}+\vec{u}_{x x}, \tilde{Q}=|\vec{u}|^{2}+\left|\vec{u}_{x}\right|^{2}$. Then the flow (38) is intrinsic and the curvature vector $\tilde{m}$ fulf ills the equation (37).

Remark 2. In the case of $n=2$, i.e., the case of $\mathbb{S}^{2}(1)$, equation (37) reduces to the scaler modified $\mathrm{CH}$ equation (4) with $\delta=-1$, which is completely integrable. In the case of $n=3$, let $u_{1}=u, u_{2}=v, m=u+u_{x x}, n=v+v_{x x}$, then system (37) reduces to

$$
\begin{aligned}
& m_{t}=\frac{1}{2}\left[\left(u^{2}+v^{2}+u_{x}^{2}+v_{x}^{2}\right) m\right]_{x}-\left(u v_{x}-v u_{x}\right) n, \\
& n_{t}=\frac{1}{2}\left[\left(u^{2}+v^{2}+u_{x}^{2}+v_{x}^{2}\right) n\right]_{x}+\left(u v_{x}-v u_{x}\right) m .
\end{aligned}
$$

In general, the multi-component system (37) can be written as

$$
m_{i, t}=\frac{1}{2} \sum_{j=1}^{n-1}\left[\left(u_{j}^{2}+u_{j, x}^{2}\right) m_{i}\right]_{x}-\sum_{j=1}^{n-1}\left(u_{i} u_{j, x}-u_{j} u_{i, x}\right) m_{j}, \quad 1 \leq i \leq n-1, \quad m_{i}=u_{i}+u_{i, x x} .
$$

Case 2. $\vec{h}=\vec{u}_{x}, \vec{k}=\vec{m}=\vec{u}-\vec{u}_{x x}$. In this case, the tangent velocity $f$ is given by

$$
f=\partial_{x}^{-1}\left\langle\vec{u}-\vec{u}_{x x}, \vec{u}_{x}\right\rangle=\frac{1}{2} \sum_{i=1}^{n-1}\left(u_{i}^{2}-u_{i, x}^{2}\right)+c_{1} \equiv \frac{1}{2} Q+c_{1},
$$

where $c_{1}$ is an integration constant. Substituting (39) with $c_{1}=1$ into (35) and noting that

$$
\left(\partial_{x}^{-1}\left(\vec{k}_{x} \wedge \vec{h}\right)\right) \vec{k}=-\left(\vec{u} \wedge \vec{u}_{x}\right) \vec{m}-\left(\vec{u}_{x} \wedge \vec{m}\right) \vec{m}=-\left(\vec{u} \wedge \vec{u}_{x}\right) \vec{m}-\langle\vec{m}, \vec{m}\rangle \vec{u}_{x}+\frac{1}{2} Q_{x} \vec{m},
$$

we obtain the multi-component modified $\mathrm{CH}$ equation

$$
\vec{m}_{t}=\frac{1}{2}(\vec{m} Q)_{x}-\left(\vec{u} \wedge \vec{u}_{x}\right) \vec{m}+2 \vec{u}_{x}, \quad \vec{m}=\vec{u}-\vec{u}_{x x} .
$$

Thus we have proved the following result. 
Theorem 3. Assume that curves $\gamma(x, t)$ on the sphere $\mathbb{S}^{n}(1)(n \geq 1)$ are governed by the flow

$$
\gamma_{t}=\left(\frac{1}{2} Q+1\right) e_{1}+\sum_{j=1}^{n-1} u_{j, x} e_{j+1}
$$

where $\left\{e_{1}, e_{2}, \ldots, e_{n}\right\}$ is the natural frame of the curves $\gamma(x, t),\left(u_{1}, u_{2}, \ldots, u_{n-1}\right)$ is defined by the curvatures $\vec{k}=\vec{m}=\vec{u}-\vec{u}_{x x}, Q=|\vec{u}|^{2}-\left|\vec{u}_{x}\right|^{2}$. Then the flow (41) is intrinsic and the curvature vector $\vec{m}$ satisfies (40).

Case 3. $\vec{h}=\vec{u}_{x}, \vec{k}=\vec{m}=-\vec{u}_{x x}$. In this case, the tangent velocity $f$ is determined by

$$
f=\partial_{x}^{-1}\left\langle-\vec{u}_{x x}, \vec{u}_{x}\right\rangle=-\frac{1}{2}\left|\vec{u}_{x}\right|^{2}+c_{2},
$$

where $c_{2}$ is an integration constant. It is inferred from (30) and (31) that

$$
\vec{\xi}=\left(\frac{1}{2}\left|\vec{u}_{x}\right|^{2}+1-c_{2}\right) \vec{u}_{x x}, \quad \Theta_{x}=0
$$

Setting $\Theta=0, c_{2}=1$ and $\vec{v}=\vec{u}_{x}$, we arrive at the multi-component short-pulse equation

$$
\vec{v}_{x t}+\frac{1}{2}\left(|\vec{v}|^{2} \vec{v}_{x}\right)_{x}+\vec{v}=0 .
$$

Hence we could prove the following result.

Theorem 4. Assume that curves $\gamma(x, t)$ on the sphere $\mathbb{S}^{n}(1)(n \geq 1)$ are governed by the flow

$$
\gamma_{t}=\left(1-\frac{1}{2}\left|\vec{u}_{x}\right|^{2}\right) e_{1}+\sum_{j=1}^{n-1} u_{j, x} e_{j+1}
$$

where $\left\{e_{1}, e_{2}, \ldots, e_{n}\right\}$ is the natural frame of the curves $\gamma(x, t),\left(u_{1}, u_{2}, \ldots, u_{n-1}\right)$ is defined by the curvatures $\vec{k}=\vec{m}=-\vec{u}_{x x}$. Then the flow (43) is intrinsic and the curvature vector $\vec{v}=\vec{u}_{x}$ satisfies (42).

The scalar equation of (42) was derived by Schäfer and Wayne [52] as a model for the propagation of ultra-short light pulses in silica optical fibers, which is also an approximation of nonlinear wave packets in dispersive media in the limit of few cycles on the ultra-short pulse scale.

It is worth noting that, analogous to the system (37), there is another version of multicomponent generalization of the modified Camassa-Holm equation (4) with $\delta=1$

$$
\vec{m}_{t}=\frac{1}{2}(\vec{m} Q)_{x}-\left(\vec{u} \wedge \vec{u}_{x}\right) \vec{m}, \quad \vec{m}=\vec{u}-\vec{u}_{x x}
$$

(where $Q=|\vec{u}|^{2}-\left|\vec{u}_{x}\right|^{2}$ ). However, in contrast to the system (37) arising from the compact Riemannian symmetric space $\mathbb{S}^{n}(1)=\mathrm{SO}(n+1) / \mathrm{SO}(n)$ with positive constant curvature, the system (44) arises from the noncompact Riemannian symmetric space with negative constant curvature, i.e., the hyperbolic space $\mathbb{H}^{n}=\mathrm{SO}(n, 1) / \mathrm{SO}(n)$. The derivation to integrable curve flows is similar to that for the $\mathbb{S}^{n}(1)$ case. In fact, the Lie algebra structure corresponding to the hyperbolic space $\mathbb{H}^{n}=\mathrm{SO}(n, 1) / \mathrm{SO}(n)$ is described by

$$
\mathfrak{s o}(n, 1)=\mathfrak{h} \oplus \mathfrak{m}=\mathfrak{s o}(n) \oplus \mathbb{R}^{n}
$$


with

$$
\left(\begin{array}{cc}
0 & p^{T} \\
p & 0
\end{array}\right) \in \mathfrak{m}, \quad\left(\begin{array}{cc}
0 & 0 \\
0 & \Theta
\end{array}\right) \in \mathfrak{h}
$$

where $p \in \mathbb{R}^{n}, \Theta \in \mathfrak{s o}(n)$. The Cartan connection matrices $\hat{\omega}\left(D_{x}\right)$ and $\hat{\omega}\left(D_{t}\right)$ of the natural frame are replaced with

$$
\hat{\omega}\left(D_{x}\right)=\left(\begin{array}{ccc}
0 & 1 & \overrightarrow{0}^{T} \\
1 & 0 & -\vec{k}^{T} \\
\overrightarrow{0} & \vec{k} & O
\end{array}\right), \quad O \in \mathfrak{s o}(n-1)
$$

and

$$
\hat{\omega}\left(D_{t}\right)=\left(\begin{array}{ccc}
0 & f & \vec{h}^{T} \\
f & 0 & -\vec{\xi}^{T} \\
\vec{h} & \vec{\xi} & \Psi
\end{array}\right), \quad \Psi \in \mathfrak{s o}(n-1),
$$

for curves on $\mathbb{H}^{n}=\mathrm{SO}(n, 1) / \mathrm{SO}(n)$. Similar results can be derived for the Hyperbolic space $\mathbb{H}^{n}$. Integrability of the equations (37) and (44) are guaranteed by the following results.

Theorem 5. The systems (44) and (37) are Lax integrable, namely they admit the following $(n+1) \times(n+1)$ Lax-pair

$$
\phi_{x}=U \phi, \quad \phi_{t}=V \phi,
$$

where for (44),

$$
U=\left(\begin{array}{ccc}
0 & 1 & \overrightarrow{0}^{T} \\
1 & 0 & \lambda \vec{m}^{T} \\
\overrightarrow{0} & -\lambda \vec{m} & 0
\end{array}\right), \quad V=\left(\begin{array}{ccc}
0 & \frac{1}{2} Q+\lambda^{-2} & \lambda^{-1} \vec{u}_{x}^{T} \\
\frac{1}{2} Q+\lambda^{-2} & 0 & \lambda^{-1} \vec{u}^{T}+\frac{1}{2} \lambda Q \vec{m}^{T} \\
\lambda^{-1} \vec{u}_{x} & -\lambda^{-1} \vec{u}-\frac{1}{2} \lambda Q \vec{m} & -\vec{u} \wedge \vec{u}_{x}
\end{array}\right),
$$

and for (37),

$$
U=\left(\begin{array}{ccc}
0 & 1 & \overrightarrow{0}^{T} \\
-1 & 0 & \lambda \tilde{m}^{T} \\
\overrightarrow{0} & -\lambda \tilde{m} & 0
\end{array}\right), \quad V=\left(\begin{array}{ccc}
0 & \frac{1}{2} \tilde{Q}-\lambda^{-2} & \lambda^{-1} \vec{u}_{x}^{T} \\
-\frac{1}{2} \tilde{Q}+\lambda^{-2} & 0 & -\lambda^{-1} \vec{u}^{T}+\frac{1}{2} \lambda \tilde{Q} \tilde{m}^{T} \\
-\lambda^{-1} \vec{u}_{x} & \lambda^{-1} \vec{u}-\frac{1}{2} \lambda \tilde{Q} \tilde{m} & -\vec{u} \wedge \vec{u}_{x}
\end{array}\right) .
$$

\section{Concluding remarks}

In this paper, geometrical formulations to several multi-component integrable systems are provided. These systems are regarded as multi-component generalizations of the $\mathrm{CH}$ equation and the modified $\mathrm{CH}$ equation, which can be obtained through the tri-Hamiltonian duality approach. We showed that an integrable generalization to the nonlinear Schrödinger equation arises from a non-stretching invariant curve flow in the three-dimensional Euclidean geometry. The integrable complex $\mathrm{CH}$ equation comes from an invariant curve flow on the Möbius 2-sphere. Furthermore, we verified that multi-component generalizations to the modified $\mathrm{CH}$ equation arise naturally from the curve flows in $n$-dimensional sphere $\mathbb{S}^{n}(1)$ and the hyperbolic space $\mathbb{H}^{n}=\mathrm{SO}(n, 1) / \mathrm{SO}(n)$.

In [27], Olver, Kamran and Tenenblat have established the theory for curves in affine symplectic geometry. The curve flows in four-dimensional affine symplectic geometry were studied in [58], and an integrable three-component equation with bi-Hamiltonian structure was obtained. The theory for curves in the centro-equiaffine symplectic geometry was established in [54]. It was shown that certain invariant curve flows in the centro-equiaffine symplectic geometry yield noncommutative $\mathrm{KdV}$ equations [47]. It is still not clear that what are the dual version of these integrable equations arising from curve flows in the affine and centro-equiaffine symplectic geometries. 


\section{Acknowledgements}

The authors would like to thank the anonymous referees for constructive suggestions and comments. This work was supported by the China NSF for Distinguished Young Scholars under Grant 10925104 and the China NSF under Grants 11071278 and 60970054.

\section{References}

[1] Ablowitz M.J., Clarkson P.A., Solitons, nonlinear evolution equations and inverse scattering, London Mathematical Society Lecture Note Series, Vol. 149, Cambridge University Press, Cambridge, 1991.

[2] Anco S.C., Bi-Hamiltonian operators, integrable flows of curves using moving frames and geometric map equations, J. Phys. A: Math. Gen. 39 (2006), 2043-2072, nlin.SI/0512051.

[3] Anco S.C., Group-invariant soliton equations and bi-Hamiltonian geometric curve flows in Riemannian symmetric spaces, J. Geom. Phys. 58 (2008), 1-37, nlin.SI/0703041.

[4] Anco S.C., Hamiltonian flows of curves in $G / \mathrm{SO}(N)$ and vector soliton equations of $\mathrm{mKdV}$ and sine-Gordon type, SIGMA 2 (2006), 044, 17 pages, nlin.SI/0512046.

[5] Anco S.C., Asadi E., Quaternionic soliton equations from Hamiltonian curve flows in $\mathbb{H}^{n}$, J. Phys. A: Math. Theor. 42 (2009), 485201, 25 pages, arXiv:0905.4215.

[6] Asadi E., Sanders J.A., Integrable systems in symplectic geometry, Glasg. Math. J. 51 (2009), 5-23.

[7] Calini A., Ivey T., Marí-Beffa G., Remarks on KdV-type flows on star-shaped curves, Phys. D 238 (2009), 788-797, arXiv:0808.3593.

[8] Camassa R., Holm D.D., An integrable shallow water equation with peaked solitons, Phys. Rev. Lett. 71 (1993), 1661-1664, patt-sol/9305002.

[9] Chou K.S., Qu C.Z., Integrable equations arising from motions of plane curves, Phys. D 162 (2002), 9-33.

[10] Chou K.S., Qu C.Z., Integrable equations arising from motions of plane curves. II, J. Nonlinear Sci. 13 (2003), 487-517.

[11] Chou K.S., Qu C.Z., Integrable motions of space curves in affine geometry, Chaos Solitons Fractals 14 (2002), 29-44.

[12] Chou K.S., Qu C.Z., Motions of curves in similarity geometries and Burgers-mKdV hierarchies, Chaos Solitons Fractals 19 (2004), 47-53.

[13] Constantin A., Kolev B., Geodesic flow on the diffeomorphism group of the circle, Comment. Math. Helv. 78 (2003), 787-804, math-ph/0305013.

[14] Constantin A., Kolev B., Integrability of invariant metrics on the diffeomorphism group of the circle, J. Nonlinear Sci. 16 (2006), 109-122, arXiv:0911.5058.

[15] Doliwa A., Santini P.M., An elementary geometric characterization of the integrable motions of a curve, Phys. Lett. A 185 (1994), 373-384.

[16] Fels M., Olver P.J., Moving coframes. II. Regularization and theoretical foundations, Acta Appl. Math. 55 (1999), 127-208.

[17] Fokas A.S., On a class of physically important integrable equations, Phys. D 87 (1995), 145-150.

[18] Fuchssteiner B., Some tricks from the symmetry-toolbox for nonlinear equations: generalizations of the Camassa-Holm equation, Phys. D 95 (1996), 229-243.

[19] Fuchssteiner B., Fokas A.S., Symplectic structures, their Bäcklund transformations and hereditary symmetries, Phys. D 4 (1981), 47-66.

[20] Geng X.G., Xue B., A three-component generalization of Camassa-Holm equation with $N$-peakon solutions, Adv. Math. 226 (2011), 827-839.

[21] Goldstein R.E., Petrich D.M., The Korteweg-de Vries hierarchy as dynamics of closed curves in the plane, Phys. Rev. Lett. 67 (1991), 3203-3206.

[22] Guha P., Olver P.J., Geodesic flow and two (super) component analog of the Camassa-Holm equation, SIGMA 2 (2006), 054, 9 pages, nlin.SI/0605041.

[23] Gui G., Liu Y., Olver P.J., Qu C.Z., Wave-breaking and peakons for a modified Camassa-Holm equation, Comm. Math. Phys., to appear. 
[24] Hasimoto H., A soliton on a vortex filament, J. Fluid Mech. 51 (1972), 477-485.

[25] Hone A.N.W., Wang J.P., Integrable peakon equations with cubic nonlinearity, J. Phys. A: Math. Theor. 41 (2008), 372002, 10 pages, arXiv:0805.4310.

[26] Ivey T.A., Integrable geometric evolution equations for curves, in The Geometrical Study of Differential Equations (Washington, DC, 2000), Contemp. Math., Vol. 285, Amer. Math. Soc., Providence, RI, 2001, $71-84$.

[27] Kamran N., Olver P., Tenenblat K., Local symplectic invariants for curves, Commun. Contemp. Math. 11 (2009), 165-183.

[28] Kouranbaeva S., The Camassa-Holm equation as a geodesic flow on the diffeomorphism group, J. Math. Phys. 40 (1999), 857-868, math-ph/9807021.

[29] Langer J., Perline R., Poisson geometry of the filament equation, J. Nonlinear Sci. 1 (1991), 71-93.

[30] Li Y.Y., Qu C.Z., Shu S.C., Integrable motions of curves in projective geometries, J. Geom. Phys. 60 (2010), 972-985.

[31] Marí Beffa G., Bi-Hamiltonian flows and their realizations as curves in real semisimple homogeneous manifolds, Pacific J. Math. 247 (2010), 163-188.

[32] Marí Beffa G., Conformal analogue of the Adler-Gel'fand-Dikii bracket in two dimensions, J. Phys. A: Math. Gen. 33 (2000), 4689-4707.

[33] Marí Beffa G., Geometric realizations of bi-Hamiltonian completely integrable systems, SIGMA 4 (2008), 034, 23 pages, arXiv:0803.3866.

[34] Marí Beffa G., Hamiltonian evolution of curves in classical affine geometries, Phys. D 238 (2009), 100-115.

[35] Marí Beffa G., On completely integrable geometric evolutions of curves of Lagrangian planes, Proc. Roy. Soc. Edinburgh Sect. A 137 (2007), 111-131.

[36] Marí Beffa G., Poisson brackets associated to the conformal geometry of curves, Trans. Amer. Math. Soc. 357 (2005), 2799-2827.

[37] Marí Beffa G., Poisson geometry of differential invariants of curves in some nonsemisimple homogeneous spaces, Proc. Amer. Math. Soc. 134 (2006), 779-791.

[38] Marí Beffa G., Olver P.J., Poisson structures for geometric curve flows in semi-simple homogeneous spaces, Regul. Chaotic Dyn. 15 (2010), 532-550.

[39] Marí Beffa G., Sanders J.A., Wang J.P., Integrable systems in three-dimensional Riemannian geometry, J. Nonlinear Sci. 12 (2002), 143-167.

[40] Misiolek G., A shallow water equation as a geodesic flow on the Bott-Virasoro group, J. Geom. Phys. 24 (1998), 203-208.

[41] Musso E., Motions of curves in the projective plane inducing the Kaup-Kupershmidt hierarchy, SIGMA 8 (2012), 030, 20 pages, arXiv:1205.5329.

[42] Nakayama K., Segur H., Wadati M., Integrability and the motion of curves, Phys. Rev. Lett. 69 (1992), 2603-2606.

[43] Novikov V., Generalizations of the Camassa-Holm equation, J. Phys. A: Math. Theor. 42 (2009), 342002 , 14 pages.

[44] Olver P.J., Applications of Lie groups to differential equations, Graduate Texts in Mathematics, Vol. 107, 2nd ed., Springer-Verlag, New York, 1993.

[45] Olver P.J., Invariant submanifold flows, J. Phys. A: Math. Theor. 41 (2008), 344017, 22 pages.

[46] Olver P.J., Rosenau P., Tri-Hamiltonian duality between solitons and solitary-wave solutions having compact support, Phys. Rev. E 53 (1996), 1900-1906.

[47] Olver P.J., Sokolov V.V., Integrable evolution equations on associative algebras, Comm. Math. Phys. 193 (1998), 245-268.

[48] Pinkall U., Hamiltonian flows on the space of star-shaped curves, Results Math. 27 (1995), 328-332.

[49] Qiao Z., A new integrable equation with cuspons and W/M-shape-peaks solitons, J. Math. Phys. 47 (2006), 112701, 9 pages.

[50] Sanders J.A., Wang J.P., Integrable systems in $n$-dimensional conformal geometry, J. Difference Equ. Appl. 12 (2006), 983-995. 
[51] Sanders J.A., Wang J.P., Integrable systems in n-dimensional Riemannian geometry, Mosc. Math. J. 3 (2003), 1369-1393, math.AP/0301212.

[52] Schäfer T., Wayne C.E., Propagation of ultra-short optical pulses in cubic nonlinear media, Phys. D 196 (2004), 90-105.

[53] Sharpe R.W., Differential geometry. Cartan's generalization of Klein's Erlangen program, Graduate Texts in Mathematics, Vol. 166, Springer-Verlag, New York, 1997.

[54] Song J.F., Qu C.Z., Integrable systems and invariant curve flows in centro-equiaffine symplectic geometry, Phys. D 241 (2012), 393-402.

[55] Song J.F., Qu C.Z., Qiao Z.J., A new integrable two-component system with cubic nonlinearity, J. Math. Phys. 52 (2011), 013503, 9 pages.

[56] Tao T., Nonlinear dispersive equations. Local and global analysis, CBMS Regional Conference Series in Mathematics, Vol. 106, Amer. Math. Soc., Providence, RI, 2006.

[57] Terng C.L., Thorbergsson G., Completely integrable curve flows on adjoint orbits, Results Math. 40 (2001), $286-309$.

[58] Valiquette F., Geometric affine symplectic curve flows in $\mathbb{R}^{4}$, Differential Geom. Appl. 30 (2012), 631-641.

[59] Wang J.P., Generalized Hasimoto transformation and vector sine-Gordon equation, in SPT 2002: Symmetry and Perturbation Theory (Cala Gonone), World Sci. Publ., River Edge, NJ, 2002, 276-283.

[60] Wo W., Qu C.Z., Integrable motions of curves in $S^{1} \times \mathbb{R}$, J. Geom. Phys. 57 (2007), 1733-1755. 